\title{
Carrier Selection for Multi-commodity Flow Optimization in Cooperative Environments
}

\author{
Giacomo Liotta ${ }^{1}$, Giuseppe Stecca ${ }^{2}$, and Toshiya Kaihara ${ }^{3}$ \\ ${ }^{1}$ Department of Technology and Innovation, University of Southern Denmark, \\ Campusvej 55, DK-5230 Odense, Denmark \\ gileiti.sdu.dk \\ ${ }^{2}$ Istituto di Analisi dei Sistemi ed Informatica del Consiglio Nazionale delle Ricerche, \\ Viale Manzoni 30, 00185 Rome, Italy \\ giuseppe.stecca@iasi.cnr.it \\ ${ }^{3}$ Graduate School of System Informatics, Kobe University, 1-1, Rokkodai, Nada, \\ 657- 8501 Kobe, Japan \\ kaihara@kobe-u.ac.jp
}

\begin{abstract}
Freight transportation decisions are critical economic and environmental factors in the design and management of networked manufacturing systems at global scale. Multimodal transportation options in combination with cooperative models between transport operators and together with manufacturers can contribute to define more economically and environmentally sustainable operations. This work addresses the problem of the selection of carriers in an international production and distribution network. The aim is to minimize costs and environmental impacts of freight transport. A cooperative decision-making setting between carriers in response to transportation demand of manufacturers is adopted. An integrated optimization-simulation approach is proposed to model the process of defining the optimal combination of transportation services in a multimodal transport network. Experiments show that collaboration based on shared modal capacity between carriers can produce transport cost reduction and service level improvements.
\end{abstract}

Keywords: Supply chain management, Simulation, Optimization, Carrier selection.

\section{$1 \quad$ Introduction}

Networked production requires effective integration of production and distribution planning. This is particularly relevant for complex products consisting of multiple components to be assembled and delivered (e.g., automotive industry). It is more and more important to develop comprehensive decision methods and systems able to (i) integrate different decision levels (i.e., strategic, tactical and operational) and (ii) consider a variety of decision variables (i.e., economic, social and environmental).

This work tackles the problem of carrier selection in an international production and distribution network. A significant literature stock covers organizational aspects and problem solving issues related to production and multimodal freight transportation planning [1]. In optimization approaches to production-distribution 
planning, the development of multiple performance measures in the objective functions, e.g., cost, service level, social and environmental impacts, is required [2]. Collaboration issues in supply chains have been extensively discussed in the literature. Horizontal cooperation in supply chains and carrier selection was demonstrated to be a source of potential benefits to increase carriers' profitability or improve service quality [3]. Carrier selection often requires multicriteria approaches within which environmental and energy use concerns are significant [4]. Collaboration among supply chain actors may enable new optimized configurations of supply chain networks. Therefore, optimization and simulation, as methods largely used in supply chain problems, can be applied to investigate collaboration settings in supply chains while including multicriteria considerations. Optimization was used to address collaboration in transport [5]. Combined optimization and simulation have been applied to collaboration settings among logistics operators and customers [6].

The literature is mainly focused on the analysis of integrated production and transportation from a strategic viewpoint. In mode choice and carrier selection problems, the inclusion of environmental impacts is under-represented [4]. This paper concurrently considers (i) optimization to support strategic decisions, and (ii) simulation to support tactical and operational decisions. Additionally, $\mathrm{CO}_{2}$ emissions of freight transport are included in both optimization and simulation to address environmental sustainability. Effects of collaboration among carriers are investigated. Carriers may offer transport services in a collaborative way by aggregating themselves and operate on behalf of a multimodal transport operator. The multimodal transport operator is in charge for representing a coalition of carriers executing the transportation service and producing the multimodal document of transport [7]. The problem is how to select proper coalitions of carriers and transportation means to achieve economic and environmental performance goals. The system complexity often requires a decomposition approach and design issues can be disconnected from the tactical and operational ones. Hereinafter, we then present how (i) optimization methods can be used to design multimodal distribution, and (ii) simulation to allocate flows to connections throughout time considering randomness effects.

\section{Methodology}

The methodological approach consists of the integrated use of optimization and simulation (Fig. 1). The assignment of product flows from factories to final destinations over the multimodal transport network is a multi-commodity capacitated network design problem. A specific problem formulation taking into account cost and environmental impact of transport is presented in Section 2.1. The solution of the optimization model supports in a static way the strategic decisions about the carriers to be activated (e.g., service contracts) for each product and routes. Furthermore, the tactical-operational decisions regarding the service provision have to consider in a dynamic way further elements such as shipments from factories throughout time, frequencies of transport services, service capacity and randomness in service times. For this purpose, a discrete event simulation model is implemented (Section 2.2). 


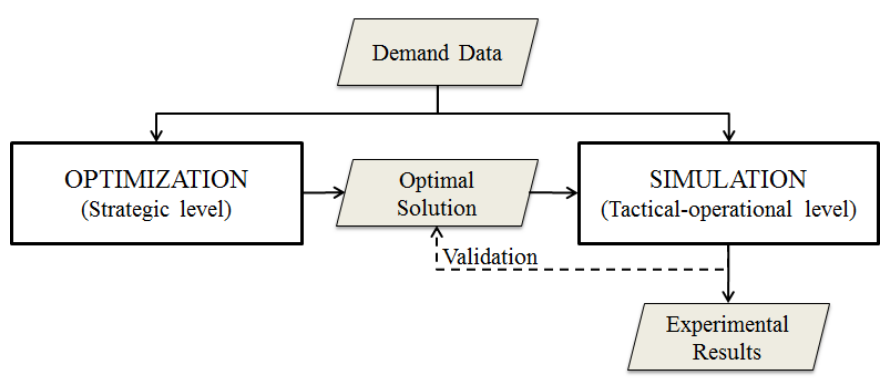

Fig. 1. Methodological approach: Optimization and simulation

First, the simulation model is validated by comparing the output to the solutions of the optimization model (dotted line in Fig. 1). Second, the simulation model is used to test alternative scenario settings by varying the capacity of scheduled services for each transport mode and, more importantly, the routing logic of product flows across the network according to a collaborative scenario setting between carriers. The simulation model processes transport demand data and solutions of the optimization model. It allows the evaluation of additional parameters and performance measures that is hard to embed in the optimization model, e.g., service frequency and times.

\subsection{Optimisation Model and Sustainability Factors}

Multimodality is modelled as an aspect of the classical multi-commodity flow problem. Gendron et al. [8] model a multimodal transportation problem as a multicommodity capacitated network design problem defined on a directed graph $G=(N$, $A$ ). Stecca et al. [9] add bill of material constraints and environmental sustainability considerations. In the model hereinafter presented, carriers can cooperate by offering a composite transportation service offered by means of a coalition. To each transport service an arc $(i, j)$ is associated between a pair of nodes. Each transportation service may be operated by different carriers belonging to a predefined set of carriers $M$. The optimization model considers transportation and $\mathrm{CO}_{2}$ emission costs. Fixed, routing costs and coalition set-up costs are also included. The problem is to select the most effective coalition to which transportation services can be outsourced. For each node $i$ $\in N$ let $\Delta^{+}(i)$ be the nodes $j \in N$ such that $(i, j) \in A$ and $\Delta^{-}(i)$, the nodes $j \in N$ such that $(j, i) \in A$ and $K$ be the set of the final products. Lead times are defined as $\left[a_{i}, b_{i}\right]$ for each $i \in N$ while $[E D, D D]$ is the earliness and lateness of the problem; it bounds the time of start and end of production and transport operations. Let moreover $s_{i}$ the service time at node $i$, for each $i \in N ; t_{i j k}$ the time needed to traverse an $\operatorname{arc}(i, j) \in A$ for shipment $k ; l t s_{k}$ is expression of inventory in time units. The model considers alternative sources for final products. To each shipment (final product) $k \in K$ it is associated a subset of alternative origin nodes $O^{k} \subset N$ in which the shipment can be produced with $O=\cup O^{k} . D^{k} k \in K$ is the set of destination nodes for shipment $k$, while $q_{d}$ is the quantity of shipment $k$ to be shipped in destination $d \in D^{k}, I^{k}$ are the subsets of intermediate nodes for shipment $k$ with $I=\cup I^{k}$. For each node $i \in O=\cup_{k \in K} O^{k}$, a production cycle time for node $i$ is defined as $r_{i} ; m \in M$ defines the generic carrier while $l \in L$ represents a coalition of carriers. Coalitions are modelled in the following 
way. The coefficient $b_{l m}$ of the matrix $b[L][M]$ is equal to 1 if the carrier $l$ is inserted in the coalition $m, 0$ otherwise. A binary variable $y_{m}$ will store which coalition will be selected. A carrier can be part of at least one coalition. The parameter $a_{i j l}$ is used to model which arc a carrier can operate on. if $a_{i j l}=1$, the carrier $l$ can operate on the arc $(i, j), 0$ otherwise. The cost parameters of the model are: $f c_{m}$, the fixed coalition set-up cost for coalition $m$; $f r_{i j}$ the fixed routing cost for $\operatorname{arc} \operatorname{arc}(i, j) \in A ; c_{i j}$, the transportation price required per unit of shipments transported along arc $(i, j) \in A ; \alpha$, the cost of a tonne of $\mathrm{CO}_{2}$ emission. Capacity parameters are: $C_{i j}$, the capacity of arcs $(i, j) \in A ; C P_{i k}$, the production capacity of node $i$ for product $k$. Other parameters are: $B i g M$, a large constant; $w s u_{k}$; the weight of a unit of shipments of $k$ in tonnes; $e_{i j k}$, emission factors where $(i, j) \in A$ and $k \in K$ in tonnes of $\mathrm{CO}_{2}$ for unit of shipment $k$. The variables of the problems are: $y_{m}, m \in M$, a binary variable defining whether the coalition $m$ is selected (1) or not $(0) ; u_{i j k},(i, j) \in A, k \in K$ binary variable equal to 1 if shipment $k$ is routed through arc $(i, j), 0$ otherwise; $x_{i j k},(i, j) \in A, k \in K$ the quantity of shipment $k$ routed through arc $(i, j) ; w_{i k}, i \in N, k \in K$ the arrival time at node $i$ for shipment $k$. The model can be formulated as follows:

$$
\min \sum_{m \in M} f c_{m} y_{m}+\sum_{k \in K} \sum_{(i, j) \in A} w s u_{k} c_{i j} x_{i j k}+\sum_{k \in K} \sum_{(i, j) \in A} f r_{i j k} u_{i j k}+\alpha \sum_{(i, j) \in A} \sum_{k \in K} w s u_{k} e_{i j} x_{i j k}
$$

subject to

$$
\begin{gathered}
x_{i j k} \leq \sum_{l \in L m \in M} \sum_{i j l} b_{l m} y_{m} B i g M \quad \forall(i, j) \in A, k \in K \\
\sum_{m \in M} b_{l m} y_{m} \leq 1 \quad \forall l \in L \\
\sum_{i \in O(k)} \sum_{j \in \Delta^{+}(i)} x_{i j k}=\sum_{d \in D^{k}} q_{d} \quad \forall k \in K \\
\sum_{i \in \Delta^{-}(d)} x_{i d k}=q_{d} \quad \forall d \in D^{k}, k \in K \\
\sum_{j \in \Delta^{+}(i)} x_{i j k}-\sum_{j \in \Delta^{-}(i)} x_{j i k}=0, \quad \forall k \in K, \forall i \in I^{k} \\
r_{k} l t s_{k}+w_{i k}+s_{i}+t_{i j k}-w_{j k} \leq\left(1-u_{i j k}\right) B i g M \\
\forall k \in K, \forall(i, j) \in A \\
E D \leq w_{i k} \leq D D, \quad \forall k \in K, \forall i \in\{O, D\} \\
x_{i j k} \leq C_{i j} u_{i j k} \forall(i, j) \in A, k \in K \\
\sum_{i \in O(k)} \sum_{j \in \Delta^{+}(i)} x_{i j k} \leq C P_{i k} \forall i \in N, \forall k \in K \\
u_{i j k} \in\left\{0, w_{i k} \geq 0, \quad \forall k \in K, \forall(i, j) \in A, y_{m} \in\{0,1\}, \forall m \in M\right.
\end{gathered}
$$


The objective function (1) consists of a first term considering the coalition set-up costs. The second and third terms consider, respectively, variable and fixed transportation costs of each travelled arc while the fourth one the $\mathrm{CO}_{2}$ emission cost. Constraint (2) links the flow variables to the service operated by the carriers of the selected coalitions. Constraint (3) imposes the carriers to belong to a single selected coalition. Constraints (4), (5) and (6) are flow constrains applied to multicommodity flow. Expressions (7) and (8) define time constraints, while functions (9) and (10) define capacity constraints and expression (11) the decision variables.

\subsection{Simulation Model}

The simulation model reproduces the sources of finished products with related quantities as well as the product routings over the transport network served by the available carriers. Freight transport modes are road, rail and sea. For each transport operator a fixed cost component is included while, for each service provided, the variable transport costs as well as the $\mathrm{CO}_{2}$ emissions and related costs are calculated. Final market destinations are also modeled. The model is implemented in the simulation modeling framework Simio (Simio LLC). A screenshot of the model is presented in Fig. 2. The implemented model, loaded with the same transport demand data and solution of the optimization model, runs throughout a simulation time horizon of 52 weeks. 50 replications are made. The simulation model operates with a deviation of $0.1 \%$ in terms of total output (i.e., products delivered to final market destinations) and $0.06 \%$ in terms of total $\mathrm{CO}_{2}$ emissions w.r.t. optimization. Half widths (95\% confidence interval) of values of main measures analyzed in validation and further experiments are negligible. The model is then valid for the study purpose.

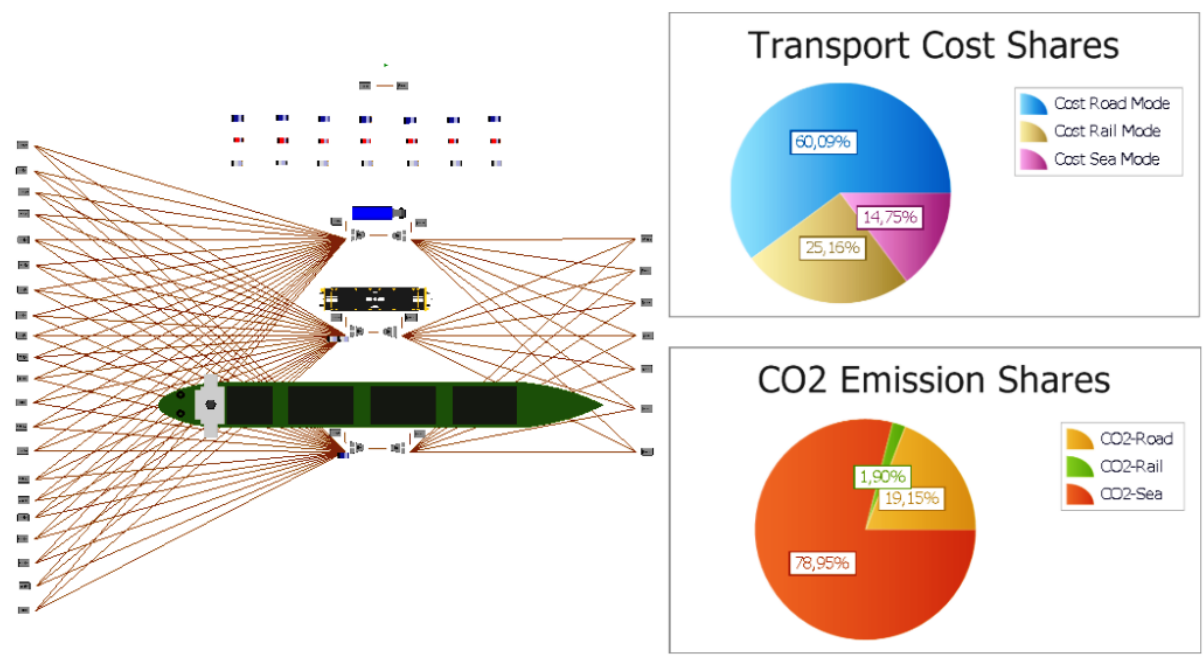

Fig. 2. Screenshot of the simulation model 


\section{Transportation Service Assignment}

The optimization model considers the selection of coalitions to deliver the transportation service but no details on how the coalition has to provide the service over the arcs are given. A clearing procedure is required for the detailed allocation of transportation services. The procedure is executed by the simulation model. As a preliminary approach, a coalition including a set of carriers operating over the multimodal network can share the transport capacity for each transport mode over the routes connecting factories to market destinations. Product shipments are then routed over the network of services according to the distance from the final destinations. So doing, potential cost and territorial leadership of single carriers can be exploited. The collaborative setting is tested in simulation experiments.

\subsection{Scenario Description and Computational Results}

The scenario used for the optimization consists of three product types (sales data are generated starting from the statistics of ACEA, http://www.acea.be/statistics) and seven carriers which can serve seven market destinations. Transport demand is equal to 705,250 tonnes/year. Three carriers provide road transport services; two carriers offer rail transport while two carriers sea transport. Road, rail and sea transport costs are equal to, respectively, 0.14, 0.11 and $0.009 \mathrm{EUR} / \mathrm{tkm}^{1} . \mathrm{CO}_{2}$ emissions of the road, rail and sea transport are equal to $93.1,17.4$ and $101 \mathrm{gCO}_{2} / \mathrm{tkm}$, respectively ${ }^{2} . \mathrm{CO}_{2}$ emission cost is $11.07 \mathrm{EUR} /$ tonne $\mathrm{CO}_{2}(\$ 15)^{3}$.

The simulation model makes use of the same demand data, parameters and number of activated carriers generated by the optimizer in the optimal solution. The simulation model is tested in two main scenario settings: (i) a baseline scenario not including the collaboration and (ii) a collaborative scenario modeling a coalition consisting of all the available transport operators. The studied case assumes distance ranges included in a European network. Basic simulation parameters for baseline and collaborative scenarios are presented in Table 1. The total cost of transport includes fixed costs for each transport operator, variable costs as well as $\mathrm{CO}_{2}$ emissions costs. In the collaborative scenario, products are routed across the network (allocated to services) according to the following distances from the final destinations: up to 700 $\mathrm{Km}$ (road mode), between 700 and 1,400 Km (rail), longer than 1,400 Km (sea).

Table 1. Simulation parameters

\begin{tabular}{lllll}
\hline Transport services & Service times (hours) & \multicolumn{2}{l}{ Frequency (services/week) } & Capacity (ton./serv.) \\
& & Baseline & Collaborative & \\
\hline Road transport & Triangular $(3,5,8)$ & 56 & 84 & 250 \\
Rail transport & Triangular $(12,17,24)$ & 14 & 28 & 450 \\
Sea Transport & Triangular $(18,23,30)$ & 7 & 14 & 900 \\
\hline
\end{tabular}

\footnotetext{
${ }^{1}$ http: / / ec. europa.eu/ten/transport/studies / doc/compete/compete _report_en.pdf

2 http: / / www . developpement-durable.gouv.fr/IMG/pdf/Information _CO2_ENG_Web-2.pdf

3 http: / /www. oecd.org/env/cc/40633555.pdf
} 
Computational results are presented in Table 2. Computational results of the optimization show that the model can be effectively used to select the proper coalition. Test instances are built by comparing a baseline situation without collaboration to a scenario considering coalitions in which each coalition is able to cover all the modal transports. The baseline situation is used to validate the simulation model. The coalition covering all the modal transports is then passed to the validated simulation model for detailed experiment of the collaborative setting.

Table 2. Computational results of the optimization-simulation approach

\begin{tabular}{llll}
\hline Transport services & Optimization & $\begin{array}{l}\text { Simulation - } \\
\text { Baseline }\end{array}$ & $\begin{array}{l}\text { Simulation - } \\
\text { Collaborative }\end{array}$ \\
\hline Total transport costs (EUR) & $57,149,206$ & $57,144,317$ & $37,697,940$ \\
$\mathrm{CO}_{2}$ emissions (tonnes) & 73,460 & 73,416 & 76,776 \\
Total output delivered (tonnes) & 705,250 & 704,550 & 705,050 \\
Road transport & & & \\
Tonnes Km carried (tkm) & $282,537,000$ & $282,537,000$ & $157,921,250$ \\
Avg. number in station (tonnes) & - & 20,325 & 48,356 \\
Avg. time in station (weeks) & - & 5.5 & 7.0 \\
Rail transport & & & \\
Tonnes Km carried (tkm) & $112,305,750$ & $112,294,300$ & $83,981,700$ \\
Avg. number in station (tonnes) & - & 60,656 & 3,986 \\
Avg. time in station (weeks) & - & 15.2 & 2.40 \\
Sea transport & & & \\
Tonnes Km carried (tkm) & $447,543,250$ & $447,115,550$ & $600,118,950$ \\
Avg. number in station (tonnes) & - & 134,573 & 46,718 \\
Avg. time in station (weeks) & - & 22.8 & 9.2 \\
\hline
\end{tabular}

The related experiments highlight a reduction in transport cost by $34 \%$. However, an increase in $\mathrm{CO}_{2}$ emissions by $4.6 \%$ w.r.t. the baseline scenario (deviation between output delivered equal to $0.07 \%$ ) can be observed. These effects can be justified by the shares of tonnes $\mathrm{km}(\mathrm{tkm})$ carried by each transport mode. In the collaborative scenario, remarkable reductions in tkm of the road mode $(-44.1 \%)$ and rail mode ($25.2 \%)$ are compensated by an increase in tkm transported through the sea mode $(+34.2 \%)$. On the other hand, the baseline scenario relies on a lower number of available carriers not allowed to share transport capacity on modal routes. The collaboration, which is ruled by the distance-based allocation of transport orders, produces positive effects on overall transport costs but also a slight increase in $\mathrm{CO}_{2}$ emissions due to the specific transport mode characteristics. In the road mode transport operators carry larger quantities across shorter distances w.r.t. the baseline scenario. Conversely, lower product quantities are carried across longer routes in the rail and sea modes. Furthermore, the collaboration has very positive effects on intransit inventory and service levels throughout the transport chain: an improvement in the sum of product quantities waiting for transportation and an average decrease in the related times can be observed. Lower workload and higher frequency of transport 
services contribute to these performance impacts. Lower levels of stored products across the transport network may also entail positive impacts on $\mathrm{CO}_{2}$ emissions due to lower risks of excess production and product obsolescence.

\section{Concluding Remarks and Future Research}

This work aims to contribute to the solution of complex freight transportation problems which have to simultaneously address (i) strategic transport decisions and their operationalization as well as (ii) the minimization of costs and environmental impacts in an international production and multimodal distribution network. Collaboration between carriers is tested in the form of coalitions represented by transport operators. Collaboration based on shared modal capacity between carriers can produce a global reduction in costs and in-transit inventory as well as service level improvements. In the studied scenario, it however seems that environmental performance does not benefit from the tested collaboration mechanism. This last result suggests that more environmentally sustainable transport decisions could be also guided, e.g., by defining at policy level proper cost structures related to $\mathrm{CO}_{2}$ emissions that transport operators should consider. $\mathrm{CO}_{2}$ emissions of production operations are out of the scope of this work. Future works will explore multicriteria approaches as well as refinements of collaboration mechanisms and incentives.

Acknowledgments. University of Southern Denmark and National Research Council of Italy use Simio simulation software under a grant from Simio LLC (www.simio.com).

\section{References}

1. SteadieSeifi, M., Dellaert, N.P., Nuijten, W., Van Woensel, T., Raoufi, R.: Multimodal Freight Transportation Planning: a Literature Review. Eur. J. Oper. Res. 233, 1-15 (2014)

2. Fahimnia, B., Farahani, R.Z., Marian, R., Luong, L.: A Review and Critique on Integrated Production-Distribution Planning Models and Techniques. J. Manuf. Syst. 32, 1-19 (2013)

3. Cruijssen, F., Cools, M., Dullaert, W.: Horizontal Cooperation in Logistics: Opportunities and Impediments. Transport. Res. E-Log. 43, 129-142 (2007)

4. Meixell, M.J., Norbis, M.: A Review of the Transportation Mode Choice and Carrier Selection Literature. The International Journal of Logistics Management 19, 183-211 (2008)

5. Frisk, M., Göthe-Lundgren, M., Jörnsten, K., Rönnqvist, M.: Cost Allocation in Collaborative Forest Transportation. Eur. J. Oper. Res. 205, 448-458 (2010)

6. Confessore, G., Corini, D., Stecca, G.: A Computational Method for Pricing of Delivery Service in a Logistics Network. Int. J. Prod. Res. 46, 1231-1242 (2008)

7. Van-Nes, R.: Design of Multimodal Transport Networks: A Hierarchical Approach. Delft University Press, Delft (2002)

8. Gendron, B., Crainic, T.: Frangioni, A.: Multicommodity Capacitated Network Design. In: Sansò, B., Soriano, P. (eds.) Telecommunications Network Planning, pp. 1-19. Kluwer, Boston (1999)

9. Stecca, G., Liotta, G., Kaihara, T.: A Model to Realise Sustainability in Networked Production and Transportation. In: Camarinha-Matos, L.M., Scherer, R.J. (eds.) PRO-VE 2013. IFIP AICT, vol. 408, pp. 559-568. Springer, Heidelberg (2013) 